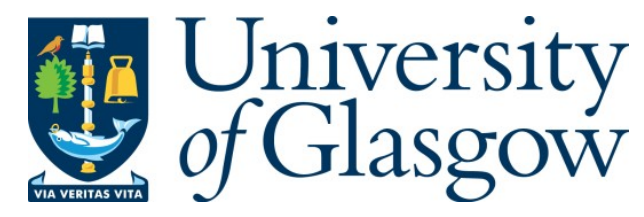

Phillips, D. B., Lee, M. P., Speirits, F. C., Barnett, S. M ., Simpson, S. H., L avery, M. P. J., Padgett, M . J . , and Gibson, G. M . (2014)Rotational $D$ oppler velocimetry to probe the angular velocity of spinning microparticles. Physical Review A: A tomic, Molecular and Optical Physics, 90 (1). 011801. ISSN 1050-2947

Copyright @ 2014 The A merican Physical Society

http://eprints.gla.ac.uk/94875/

Deposited on: 07 July 2014

Enlighten - Research publications by members of the University of Glasgow http://eprints.gla.ac.uk 


\title{
Rotational Doppler velocimetry to probe the angular velocity of spinning micro-particles
}

\author{
D. B. Phillips, ${ }^{1, *}$ M. P. Lee ${ }^{1, *}$ F. C. Speirits,${ }^{1}$ S. M. Barnett,${ }^{1}$ \\ S. H. Simpson, ${ }^{2}$ M. P. J. Lavery, ${ }^{1}$ M. J. Padgett, ${ }^{1}$ and G. M. Gibson ${ }^{1, \dagger}$ \\ ${ }^{1}$ SUPA, School of Physics and Astronomy, University of Glasgow, Glasgow, G12 8QQ, UK. \\ ${ }^{2}$ H. H. Wills Physics Laboratories, University of Bristol, Bristol, BS8 1TL, UK.
}

\begin{abstract}
Laser Doppler velocimetry is a technique used to measure linear velocity, ranging from that of exhaust gases to blood flow. A rotational analogue of laser Doppler velocimetry was recently demonstrated, using a rotationally symmetric interference pattern to probe the angular velocity of a spinning object. In this work, we demonstrate the use of a diffraction limited structured illumination pattern to measure the angular velocity of a micron sized particle trapped and spinning at tens of $\mathrm{Hz}$ in an optical trap. The technique requires no detailed knowledge of the shape of the particle, or the distribution of scatterers within it, and is independent of the particle's chirality, transparency and birefringence. The particle is also subjected to Brownian motion, which complicates the signal by affecting the rotation rate and the rotation axis. By careful consideration of these influences, we show how the measurement is robust to both, representing a new technique with which to probe the rotational motion of micro-scale particles.
\end{abstract}

Beyond the straight-forward use of the Doppler shift in the measurement of the linear velocity of an approaching object, it is also possible to exploit the Doppler shift to measure its transverse velocity, v. Key to this latter application is that an illuminating beam diffusely scatters from the translating object. Any light scattered away from the angle of reflection undergoes a change in its transverse momentum. Scattered light detected at such an angle has a frequency shift determined by the transverse velocity of the scattering surface. For example, if the particle is illuminated at incident angle $\alpha$ and viewed at normal incidence, then the Doppler shift is reduced from the linear case and is given by $\Delta \omega=\sin \alpha k_{o} \mathrm{v}$, where $k_{o}=2 \pi / \lambda$. If two beams are incident at $\pm \alpha$, as shown in Fig. 1a, the Doppler shifts are in opposite directions and interference between the two beams at the detector gives a time-dependent intensity modulation in the back-scattered light of $\omega_{\text {mod }}=2|\Delta \omega|=2 \sin |\alpha| k_{o} \mathrm{v}$. This technique is commonly referred to as laser Doppler velocimetry [1].

Exactly the same expression for the intensity modulation in the back-scattered light can be obtained by considering the scattering particle translating through the fringe pattern created by the interference of the two plane waves incident at $\pm \alpha$. Whereas the Doppler shift is an explanation of this effect in the frequency domain, the periodic modulation in scattered light as the particle traverses high and low intensity regions of the fringe pattern (where the fringe spacing is given by $\lambda / \sin |\alpha|$ ) is an explanation of the same phenomenon in the time domain.

A rotational analogue to linear Doppler velocimetry was recently demonstrated [2]: the angular velocity of a

\footnotetext{
* These authors have contributed equally to this work

${ }^{\dagger}$ Electronic address: Graham.Gibson@glasgow.ac.uk
}

spinning disk was measured by observing the modulation in scattered light as it span through a rotationally symmetric 'petal' shaped pattern created by the interference of two beams carrying orbital angular momentum (OAM) of opposite helicity [3]. As in the linear case, this method can also be interpreted in two ways. In the time domain, consideration of the motion of scattering centres around a petal pattern of rotational symmetry $N=2 \ell$ [4], where $\ell$ is the azimuthal mode number defining the helicity of the beams, results in a modulation in scattered light of angular frequency $\omega_{\text {mod }}=N \Omega$, where $\Omega$ is the angular velocity of the particle. In the frequency domain, the signal is again interpreted as a beat frequency generated by the interference of red and blue Doppler shifted light at the detector [5-7]. In an analogous way to the linear Doppler shift experienced by plane wave scattering from a translating surface, a beam carrying OAM experiences a frequency shift when scattered from a rotating surface. The helical phase fronts of an OAM beam of mode $\ell$ result in a Poynting vector that is skewed with respect to the beam axis by an angle $\beta=\sin ^{-1}\left(\ell / k_{o} r\right)$, where $r$ is the radius from the beam axis [8]. Light scattered back along the beam axis from a rotating object will be shifted in frequency by $\Delta \omega=\sin \beta k_{o} \mathrm{v}=\ell \Omega$, where $\mathrm{v}=\Omega r$. Two beams with opposite values of $\ell$, undergo frequency shifts in opposite directions, and the two components interfere at the detector to give an intensity modulation of $\omega_{\text {mod }}=2|\Delta \omega|=2|\ell| \Omega=N \Omega$, equivalent to the above. This situation is shown in Fig. $1 b$.

OAM beams have also been used to measure the rotation of a chiral object [10], the tangential component of the motion of Rubidium vapour by observing the broadening of an absorption band [11], and to structure the phase profile of an illuminating beam before interfering the scattered light with a plane wave [12]. The rotational Doppler effect associated with spin angular momentum has also been used to measure rotation, by observing the 
a)

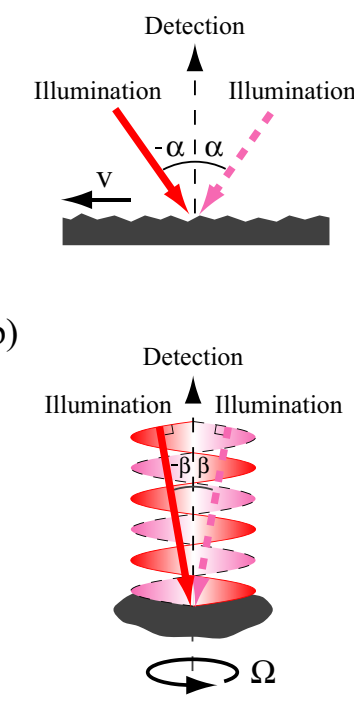

c)

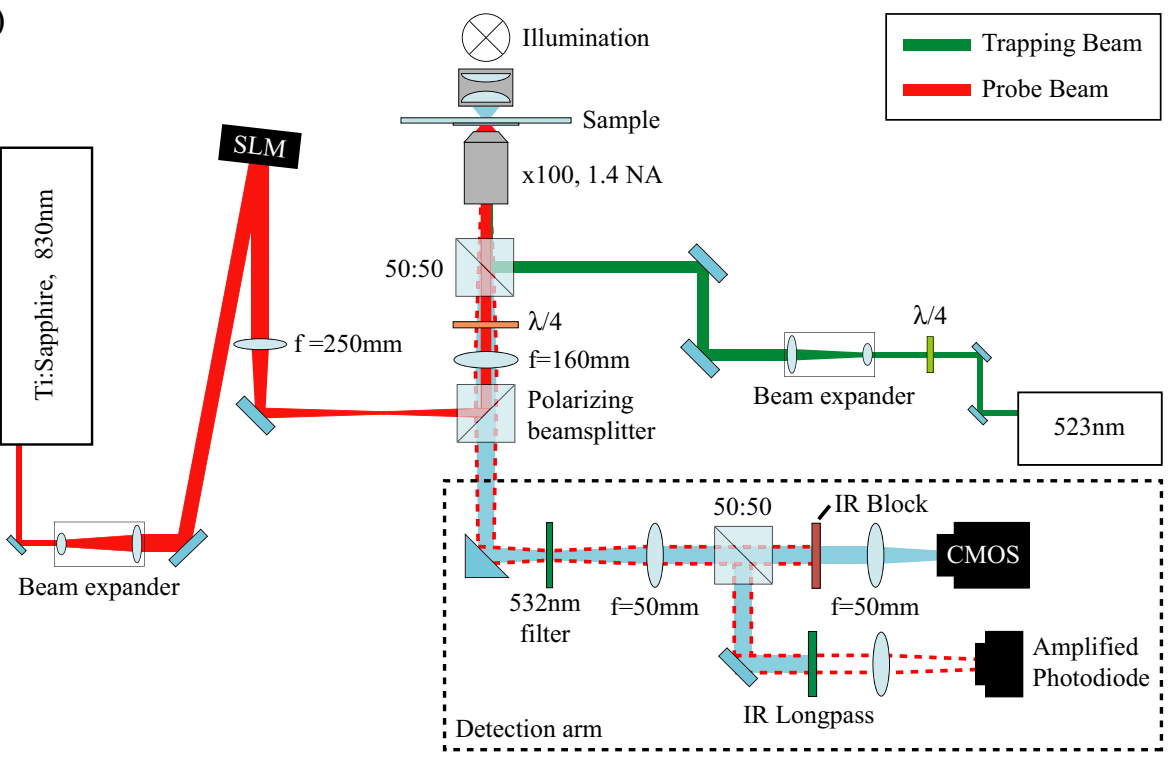

FIG. 1: (Color online). a) Laser Doppler velocimetry. Two plane waves are incident at angles $\pm \alpha$ creating interference fringes that a scattering object translates through. b) Rotational Doppler velocimetry. Two overlapping helical beams of opposite helicity $( \pm \ell)$ create a rotationally symmetric 'petal' shaped interference pattern that a scattering object rotates though. The red and pink colours indicate each overlapping beam of the same wavelength. The arrows indicate the local direction of the Poynting vector of an equivalent position on each beam. c) Experimental setup. The system is adapted from that described in [9]. A solid state laser (Laser Quantum, Opus) provides a trapping beam, which is circularly polarised using a quarter waveplate. A titanium sapphire laser $\left(\mathrm{M}^{2}\right.$, SolsTiS) provides a probe beam. The probe beam is shaped using an SLM (Boulder Nonlinear Systems, XY-Series), and focussed into the sample to the same plane as the trapped calcite particle, with the optical axis collinear with the rotation axis of the particle. In the detection arm a high-speed CMOS camera (Dalsa Genie, HM1024) is used to observe the trapped particle and an amplified silicon photodetector (Thorlabs, PDA36A-EC) monitors the intensity modulation of probe beam light that is back-scattered from the particle (shown in dashed red). A $532 \mathrm{~nm}$ block filter and an IR long pass filter prevents any unwanted light from reaching the photodetector.

change in polarisation of light transmitted through a rotating birefringent particle $[13,14]$, and change in wavelength of circularly polarised light transmitted through a gas of synchronously spinning molecules [15].

In this Letter, we demonstrate the use of a structured illumination pattern to measure the angular velocity of a micron sized particle spinning in an optical trap. We generate the pattern by shaping a Gaussian beam holographically [16] into two overlapping beams of opposite helicity that are focussed to the sample. However, the phase profile is unimportant - any method of generating a rotationally symmetric intensity pattern will work. Our technique is quite general, requiring no detailed knowledge of the shape of the particle, or the distribution of scatterers within it, and is independent of the particle's chirality, transparency and birefringence. The only conditions are that the particle must scatter light anisotropically (i.e., it is not a homogenous sphere), be rigid on the timescale of the measurement, and be rotating about a reasonably well defined axis. We explore the fundamental limits of the technique, as the particle size is of the same scale as the diffraction limited beam waist. In addition, the particle is also subjected to Brownian motion, which affects both the rotation rate and the rotation axis, mak- ing the measurement more challenging. Nevertheless, we show that a rotationally symmetric structured illumination pattern can be used to unambiguously recover information on the micro-scale particle's rotation rate.

Experimental set-up. Figure 1c shows a schematic of the experiment, consisting of an inverted microscope integrated with two optical tweezers of different wavelengths. We trap and drive the rotation of a birefringent calcite particle at a rate of $20-30 \mathrm{~Hz}$ using a $50 \mathrm{~mW}$ circularly polarised single beam optical trap (shown in green) [17]. In order to make a measurement of the rotation rate of the particle independently from the driving beam, we also illuminate it with a lower power (such that it has a negligible effect on the rotation rate, estimated to be less than $1 \mathrm{~mW}$ reaching the sample), structured probe beam (shown in red). Figure 2a shows an example of the intensity cross section through these patterns for different values of $N$. Light from the probe beam is scattered from the rotating particle back onto the optical axis and imaged onto a photodetector.

Measurements. When trapped, the particle experiences a torque from the circularly polarized trapping 
a)
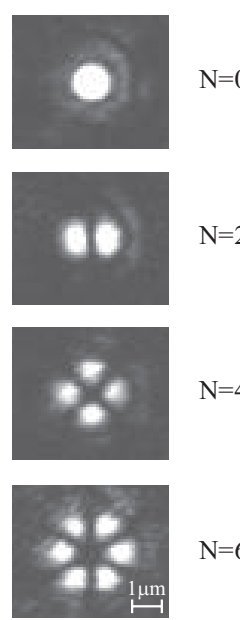

b)

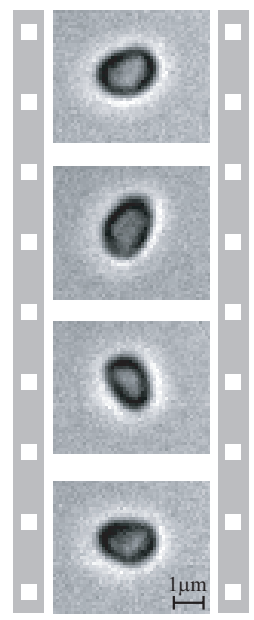

c)

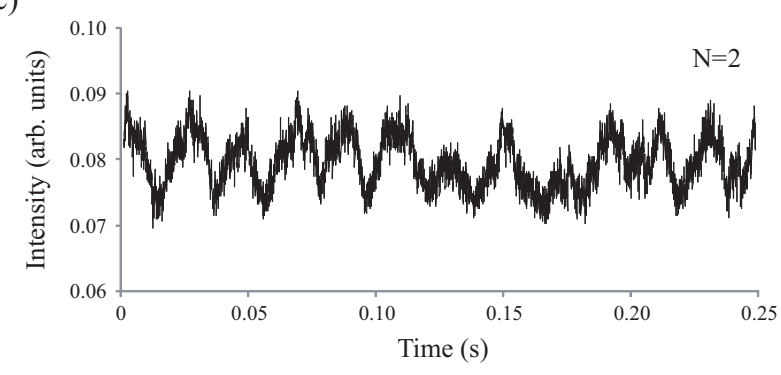

FIG. 2: a) 'Petal' shaped intensity patterns of the probe beams when focussed on the coverglass. b) Selected images of the calcite particle rotating in the optical trap. c) A small segment of the time varying signal measured by the photodetector for an $N=2$ illumination pattern. The beat frequency at $N \Omega$ can be seen (period $\sim 0.02 \mathrm{~s}$ ), along with noise introduced by Brownian motion.

beam, hydrodynamic drag from the surrounding fluid, and a stochastic torque due to Brownian motion. The balance of these effects results in the particle rotation rate fluctuating about its mean value. To validate our measurements, we also measure the rotation rate of the particle by tracking its orientation from a sequence of highspeed camera images (10,000 images recorded at $2786 \mathrm{~Hz}$, sample images shown in Fig. 2b), using the methods described in [18]. The mean rotation rate was measured as $25.6 \mathrm{~Hz}$, and found to vary by a standard deviation of $1.1 \mathrm{~Hz}$ owing to stochastic effects.

The calcite particle was positioned approximately $20 \mu \mathrm{m}$ from other surfaces to reduce the amount of probe beam light scattered from the coverslip to the detector. Figure $2 \mathrm{c}$ shows a segment of the time varying signal measured by the photodetector for an $N=2$ probe beam. 30 data runs, each consisting of 20,000 measurements at a sampling rate of $20 \mathrm{kHz}$, were recorded for illumination patterns consisting of $N=0$, 2,4 and 6 . In each case the data was Fourier transformed to produce a power spectrum, with the average power spectrum for each petal number $N$ shown in Fig. 3 .
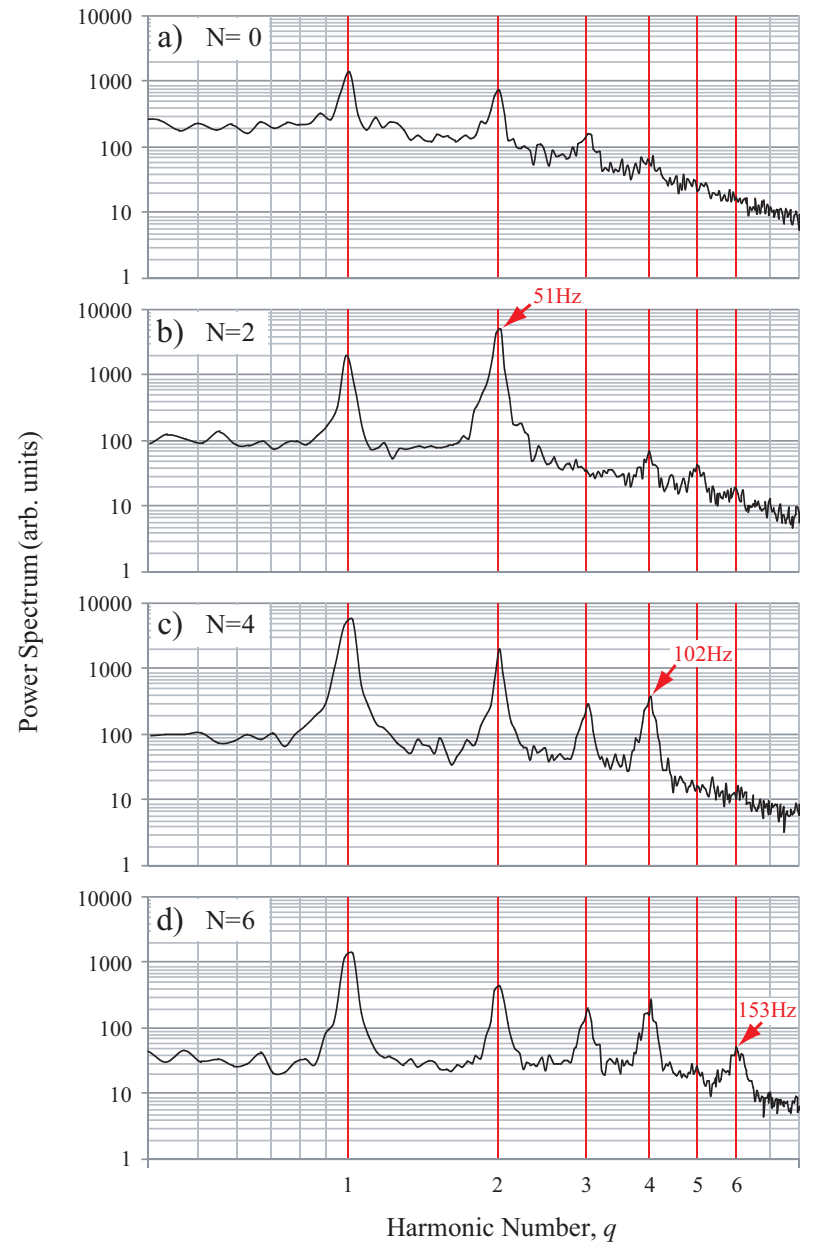

FIG. 3: (Color online). Power spectra for probe beams consisting of $N=0,2,4$ and 6 . The fundamental rotation frequencies for a), b), c) and d) are $23.9,25.3,25.5$ and $25.5 \mathrm{~Hz}$ respectively, which compares well with the rotation rate determined using video tracking. Arrows indicate frequencies of $N \Omega$. The high on-axis intensity of the $N=0$ probe beam results in a slight reduction in the rotation rate of the particle.

Results. The average power spectra show growth in peaks corresponding to $N \Omega$ (marked with arrows), as found for the case of light scattered from a spinning disk [2]. The power spectra also feature frequencies other than at $N \Omega$ because the photodetector signal is not purely sinusoidal. There are no other resonant signals present, i.e. all pronounced peaks in the power spectra are harmonics of the particle's fundamental rotation rate, due to the fact that our system is heavily over damped. However, it is the scaling of the magnitude of the harmonic at $N \Omega$ with $N$ that provides a signature of rotational motion.

The presence of harmonics at frequencies other than $N \Omega$ (and in particular the presence of the lower harmonics we observe) is predicted by considering the rotation of the particle in the presence of Brownian mo- 
tion. First consider a particle rotating about a fixed axis. We take the particle rotation axis as the origin, which is not necessarily perfectly coincident with the centre of the illuminating light. We can expand the complex amplitude of the light illuminating the particle, $u$, as a Fourier series in cylindrical coordinates: $u(r, \phi)=\sum_{n=0}^{\infty} u_{n}(r) e^{i n \phi}$, where $r$ is the radial coordinate, $\phi$ is angular coordinate, and $n$ the mode number of the expansion. The particle will scatter this light in a spatially dependent manner. We can express the scattered field as $s(r, \phi, t)=u(r, \phi) f(r, \varphi(t))$, where $f$ is a function that depends upon the properties of the scatterer, such as its geometry and reflectivity. Function $f$ also encodes the orientation of the particle at time $t$, which is given by $\varphi(t)=\phi_{0}-\Omega t+\theta(t)$. Here $\phi_{0}$ is the initial orientation of the particle, $\Omega$ is the average angular velocity as above, and $\theta(t)$ is a zero-average fluctuating angle, driven by Brownian motion. We can also expand $f$ as a Fourier series in cylindrical coordinates, $f\left(r, \phi_{0}-\Omega t+\theta t\right)=\sum_{m=0}^{\infty} f_{m}(r) e^{i m\left[\phi_{0}-\Omega t+\theta(t)\right]}$, where $m$ is the mode number of the expansion. The measured photocurrent $i(t)$ is proportional to the intensity of the backscattered light integrated over the on-axis detector area A (here we assume $A(r)=1$ for a radius $r$ less than the radius of the detector, and 0 otherwise). Therefore, the expected frequency spectrum, $S_{N}(\nu)$, of the detector signal for light scattered by a particle illuminated with a probe beam with $N$ petals, can be found from the Fourier transform of the autocorrelation function of $i(t)$, using a diffusion model for Brownian motion [19]. This results in

$$
S_{N}(\nu)=2 T \sum_{q=0}^{\infty}|G(N, q)|^{2}\left[\frac{2 D q^{2}}{(q \Omega-\nu)^{2}+D^{2} q^{4}}\right] .
$$

Here $\nu$ is the angular frequency of the power spectrum, $T$ is the length of the measurement, $D$ is the rotational diffusion coefficient of the particle, and $q$ is the azimuthal mode number of each expansion (equivalent to the harmonic number in Fig. 3). Equation 1 indicates that we can expect peaks in the power spectrum at harmonics of the rotation rate, as observed. $G(N, q)$ contains information about the angular Fourier components of the scatterer $f$, and of the illuminating beam $u$. It encodes the weighting of each harmonic: the greater the overlap integral between angular Fourier components of $f$ and $u$, the greater the power in harmonics of those modes. Therefore, as would be expected, the shape of both the light field and the particle itself play a role in determining the spectra. By illuminating with an intensity pattern of a defined rotational symmetry $N=2|\ell|$, we effectively boost the overlap integral between the particle and the light field at mode $|\ell|$. It is because of this that we observe a significant increase in the power in peaks at $N \Omega$, shown in Figure 3. Any small aberrations in the illuminating probe beam, or slight misalignments between the rotation axis of the particle and the optical axis of the probe beam, which will occur as the particle is buffeted around by Brownian motion, act to broaden the Fourier components of $u$ (when decomposed about an origin taken as the particle's current rotation axis), and result in a spread of power into nearby harmonics. As the size of each peak is governed by the overlap integral of $u$ and $f$, even a small amount of optical power into a neighbouring mode can result in large harmonics, if the corresponding Fourier component of $f$ has a large amplitude.

Our analytical model also captures another aspect of the effect of Brownian motion on the measured spectra, given by the Lorentzian term in square brackets in Eq. 1. As the particle rotates, it experiences a stochastic Brownian torque causing the rotation rate to fluctuate with a characteristic variance. These fluctuations cause a spread in each peak of the power spectrum, an effect that becomes increasingly severe for higher frequency harmonics, as can be seen from Eq. 1. The Lorentzian term modulates the shape of each harmonic so that the width increases as $\sim q^{2}$, and hence the height decreases as $\sim q^{-2}$. We clearly observe this effect in our measured spectra in Fig. 3. Therefore, even if power is spread symmetrically into adjacent harmonics, the resultant spectral density of the peaks is asymmetric, resulting in the magnitude of harmonics higher than $N \Omega$ rapidly falling off. In our experiment, increasing $N$ also increases the diameter of the petal pattern, therefore reducing the overlap integral with the particle, and hence the power in the rotational Doppler shift peak.

The presence of the additional harmonics in the power spectra can also be understood in the frequency domain: a spread in the OAM mode content will result in a different Doppler shift for each mode, as each is incident from a different angle $\beta$. Interference between all combinations of these frequencies at the detector results in the harmonics in the power spectrum.

In summary we have shown that it is possible to use rotationally symmetric structured illumination to measure the rotation rate of a microscopic calcite particle spinning in an optical trap. As the rotational symmetry $N$ of the probe beam is varied, we observe a significant increase in power at the harmonic at $N \Omega$, and it is this scaling which provides a signature of rotational motion. We also show how additional harmonics in the power spectra arise from any small misalignments between the beam and rotation axes, and the effects of Brownian motion. We have demonstrated this measurement on a particle of five orders of magnitude smaller in size than that used in the recent demonstration of angular velocity measurement on a macro-scale spinning disk [2]. Our work shows the method to be robust to Brownian noise, and allows us to distinguish the rotation of the particle from the fluctuations arising from linear motions. As our measurements are performed using a photodetector, 
our technique may also be important in discerning the high-frequency motion (beyond video tracking rates) of particles that are trapped at low pressure where translational resonances are also present, a field attracting significant interest due to its applications to mesoscale preparation of quantum state objects [20].

[1] T. Asakura and N. Takai, Appl. Phys. 25, 179 (1981).

[2] M. P. J. Lavery, F. C. Speirits, S. M. Barnett, and M. J. Padgett, Science 341, 537 (2013).

[3] L. Allen, M. W. Beijersbergen, R. J. C. Spreeuw, and J. P. Woerdman, Phys. Rev. A 45, 8185 (1992).

[4] M. S. Soskin, V. N. Gorshkov, M. V. Vasnetsov, J. T. Malos, and N. R. Heckenberg, Phys. Rev. A 56, 4064 (1997).

[5] G. Nienhuis, Opt. Commun. 132, 8 (1996).

[6] J. Courtial, D. A. Robertson, K. Dholakia, L. Allen, and M. J. Padgett, Phys. Rev. Lett. 81, 4828 (1998).

[7] A. Belmonte and J. P. Torres, Opt. Lett. 36, 4437 (2011).

[8] J. Leach, S. Keen, M. J. Padgett, C. Saunter, and G. D. Love, Opt. Express 14, 11919 (2006).

[9] G. M. Gibson, J. Leach, S. Keen, A. J. Wright, and M. J. Padgett, Opt. Express 16, 14561 (2008).

[10] I. V. Basistiy, A. Y. Bekshaev, M. V. Vasnetsov, V. V.
Slyusar, and M. S. Soskin, JETP Lett. 76, 486 (2002).

[11] S. Barreiro, J. W. R. Tabosa, H. Failache, and A. Lezama, Phys. Rev. Lett. 97, 113601 (2006).

[12] C. Rosales-Guzmán, N. Hermosa, A. Belmonte, and J. P. Torres, Sci. Rep. 3, 2815 (2013).

[13] T. Nieminen, R. Heckenberg, and H. RubinszteinDunlop., J. Mod. Opt. 48, 405 (2001).

[14] A. I. Bishop, T. A. Nieminen, N. R. Heckenberg, and H. Rubinsztein-Dunlop, Phys. Rev. A 68, 033802 (2003).

[15] O. Korech, U. Steinitz, R. J. Gordon, I. S. Averbukh, and Y. Prior, Nat. Photon. 7, 711 (2013).

[16] R. W. Bowman, G. M. Gibson, A. Linnenberger, D. B. Phillips, J. A. Grieve, D. M. Carberry, S. Serati, M. J. Miles, and M. J. Padgett, Comput. Phys. Commun. 185, 268 (2014).

[17] M. E. J. Friese, T. A. Nieminen, N. R. Heckenberg, and H. Rubinsztein-Dunlop, Nature 394, 348 (1998).

[18] D. B. Phillips, D. M. Carberry, S. H. Simpson, H. Schäfer, M. Steinhart, R. Bowman, G. M. Gibson, M. J. Padgett, S. Hanna, and M. J. Miles, J. Opt. 13, 044023 (2011).

[19] D. MacDonald, Noise and Fluctuations: An Introduction (Wiley, 1962).

[20] Y. Arita, M. Mazilu, and K. Dholakia, Nat. Commun. 4, 2374 (2013).

Acknowledgements This research is supported by the EPSRC. M.J.P. acknowledges support from the Royal Society. 